\title{
L’acceptabilité sociale de l'énergie éolienne au Québec : quel rôle pour les élus municipaux?
}

\author{
Évariste Feurtey, Carol Saucier, Gilles Côté et Bruno Jean \\ Université du Québec à Rimouski
}

\section{INTRODUCTION}

Le développement de la filière éolienne au Québec découle d'une volonté exprimée par le gouvernement du Québec dans sa politique énergétique (2006-2015). Cette volonté s'est traduite concrètement par l'adoption d'une série de décrets ordonnant à Hydro-Québec de lancer des appels d'offres pour la production d'énergie d'origine éolienne totalisant $4000 \mathrm{MW}$.

\section{À cause des pouvoirs accordés aux municipalités, les élus municipaux interviennent dans l'implantation de la filière éolienne en élaborant et en appliquant des normes encadrant la réalisation des projets.}

La réalisation de projets éoliens est encadrée par des dispositions législatives et réglementaires dans les domaines de l'environnement et de l'aménagement du territoire. En outre, la Loi sur l'aménagement et l'urbanisme $(\mathrm{LAU})^{\mathrm{a}}$ comporte des dispositifs permettant aux municipalités et aux municipalités regroupées dans des municipalités régionales de comté (MRC) d'intervenir en matière d'aménagement et d'urbanisme sur leur territoire. À cause des pouvoirs accordés aux municipalités, les élus municipaux interviennent dans l'implantation de la filière éolienne en élaborant et en appliquant des normes encadrant la réalisation des projets. Également, les élus sont appelés à jouer un rôle important à l'occasion de la réalisation de projets spécifiques. Ces derniers sont sollicités à la fois par les promoteurs et leurs concitoyens pour intervenir dans la recherche de solutions aux problèmes suscités par l'implantation des projets et la recherche de mesures visant la maximisation des retombées pour la communauté. Également, on leur demande de jouer le rôle d'arbitre pour concilier les intérêts divergents mobilisés par la réalisation des projets.

Dans ce contexte, les Conférences régionales des Élus (CRÉ) de l'Est du Québec (Bas-SaintLaurent, Chaudière-Appalaches, Côte-Nord, et Gaspésie-Îles-de-la-Madeleine) ont sollicité des chercheurs de l'Université du Québec à Rimouski et son Unité de recherche sur le développement territorial durable et la filière éolienne dans le but de participer à l'élaboration d'un Guide de l'éolien à l'intention des élus municipaux du Québec ${ }^{\mathrm{b}}$. L'objectif visé par l'élaboration de ce guide consiste à appuyer les élus dans l'exercice de leurs responsabilités.

Sur le plan du contenu, le guide comporte plusieurs informations sur l'énergie éolienne et le contexte d'implantation de la filière au Québec (historique, étapes d'implantation d'un projet éolien, cadre institutionnel québécois et types de projets implantables au Québec). La question des impacts des projets éoliens sur les composantes de l'environnement physique et humain est également abordée. Les impacts sur le paysage, les retombées économiques des projets à l'échelle locale et l'impact du bruit des turbines sur les populations dans les secteurs limitrophes sont parmi les thèmes abordés. De plus, la notion de "l'acceptabilité sociale » est explicitée. En outre, on identifie les caractéristiques d'une démarche qui favorise l'acceptabilité sociale. Finalement, le guide vise d'abord et avant tout à informer les élus sur leurs rôles et responsabilités par rapport à l'implantation de la filière éolienne et sur son potentiel en termes de développement territorial.

Le présent article vise à donner un aperçu du Guide de l'éolien. Il sera d'abord question de la notion d'acceptabilité sociale puis des rôles et responsabilités des élus municipaux du Québec. 


\section{UNE NOUVELLE DIMENSION DU DÉVELOPPEMENT : L’ACCEPTABILITÉ SOCIALE}

La notion «d'acceptabilité sociale » est de plus en plus répandue dans le discours des acteurs sociaux qui s'y réfèrent à la fois en tant que démarche et en tant que résultat. En tant que démarche, elle est identifiée à la participation des citoyens au processus de prise de décision. En tant que résultat, elle implique l'adhésion de citoyens à la décision. La plupart des articles recensés sur le sujet ont été écrits au cours des six ou sept dernières années. Il s'agit donc d'une notion dont l'usage est récent. Toutefois, il est intéressant de constater qu'aucun des auteurs ne donne de définition de l'acceptabilité sociale à proprement parler. Plutôt, ils identifient des "facteurs constitutifs de l'acceptabilité sociale ». Ces facteurs constitutifs sont reliés à l'implantation de la filière éolienne, aux caractéristiques des projets spécifiques, aux caractéristiques $\mathrm{du}$ processus décisionnel ou aux caractéristiques du milieu social ${ }^{\mathrm{c}}$ (Figure 1).

Malgré la perception initiale positive dont bénéficie généralement la filière éolienne en tant qu'énergie verte, la réalisation de projets spécifiques suscite la controverse, voire l'opposition des populations

concernées.
Parmi les questions soulevées par les chercheurs, pourquoi, malgré la perception initiale positive dont bénéficie généralement la filière éolienne en tant qu'énergie verte, la réalisation de projets spécifiques suscite la controverse, voire l'opposition des populations concernées? D'aucuns considèrent que la montée des oppositions est la manifestation du syndrome « Pas dans ma cour» (NIMBY-Not In My Backyard) qui est associé à l'individualisme et à l'égoïsme des citoyens, préoccupés davantage par leur intérêt personnel que par l'intérêt collectif. Aujourd'hui, plusieurs recherches tendent à démontrer que cette vision des choses est réductrice de la réalité et que les causes des oppositions sont plus complexes que cette analyse le laisse croire. En outre, dans bon nombre des cas étudiés, les opposants remettent en cause la légitimité du processus décisionnel estimant que les moyens utilisés pour garantir la participation des citoyens (diffusion de l'information, possibilité de participer au débat et de donner son opinion, de participer à la décision) sont insuffisants ou inadéquats. Également, on s'en prend à l'équité de la décision parce que conduisant à une répartition inégale des avantages et des inconvénients au sein de la communauté.

Figure 1 : Facteurs constitutifs de l’acceptabilité sociale

\begin{tabular}{|c|c|}
\hline DIMENSIONS & FACTEURS CONSTITUTIFS \\
\hline \multirow{2}{*}{ Filière } & Attitude initiale \\
\cline { 2 - 2 } & Cadre institutionnel \\
\hline \multirow{2}{*}{ Projet } & Impacts \\
\cline { 2 - 2 } & Retombées \\
\cline { 2 - 2 } & Origine et contrôle local \\
\hline \multirow{2}{*}{ Processus décisionnel } & Légitimité du processus \\
\cline { 2 - 2 } Caractéristiques du milieu social & Équité de la décision \\
\cline { 2 - 2 } & Capital social \\
\hline \multirow{2}{*}{} & Historique du territoire (projet controversé) \\
\hline
\end{tabular}




\section{La répartition des avantages et des inconvénients au sein de la communauté est donc au cœur du questionnement sur l'acceptabilité sociale et d'une approche de développement territorial durable qui doit harmoniser les opportunités économiques avec les contraintes environnementales et les attentes sociales.}

Le problème de la répartition des avantages et des inconvénients se pose de façon particulière dans le contexte québécois compte tenu du modèle d'implantation de la filière retenu, lequel s'appuie sur un système d'appels d'offres au plus bas coût possible. L'application de ce modèle a favorisé la réalisation de projets de grande envergure (économie d'échelle) en milieu habité (coût de raccordement au réseau), augmentant du coup les probabilités d'impacts importants, notamment sur le paysage. La question des retombées des projets à l'échelle locale a constitué un autre enjeu des projets. L'établissement du montant des redevances versées aux individus ou aux municipalités a en effet occupé une place importante dans le débat public. D'aucuns auraient souhaité qu'on laisse une place plus grande à la production à petite échelle sous le contrôle des communautés locales. La répartition des avantages et des inconvénients au sein de la communauté est donc au cœur du questionnement sur l'acceptabilité sociale et d'une approche de développement territorial durable ${ }^{\mathrm{d}}$ qui doit harmoniser les opportunités économiques avec les contraintes environnementales et les attentes sociales.

\section{RÔLES ET RESPONSABILITÉS DES ÉLUS MUNICIPAUX DU QUÉBEC}

Les élus ont un grand rôle à jouer pour la mise en application de l'acceptabilité sociale à l'échelle locale. En tant que représentants de la population locale, l'acceptation (ou non) d'un projet éolien par la population peut être de leur ressort. Parmi les dimensions de l'acceptabilité sociale, certaines peuvent être influencées plus directement par les élus. Dans cette section, elles sont détaillées sous l'angle des Rôles et responsabilités des élus. Tel que schématisé à la figure 2, nous suggérons une approche basée sur l'information, la consultation et la concertation des populations locales en amont de la prise de décision, de même que sur la négociation et l'encadrement réglementaire ${ }^{1}$.

Figure 2 : Schéma conceptuel de la prise de décision locale

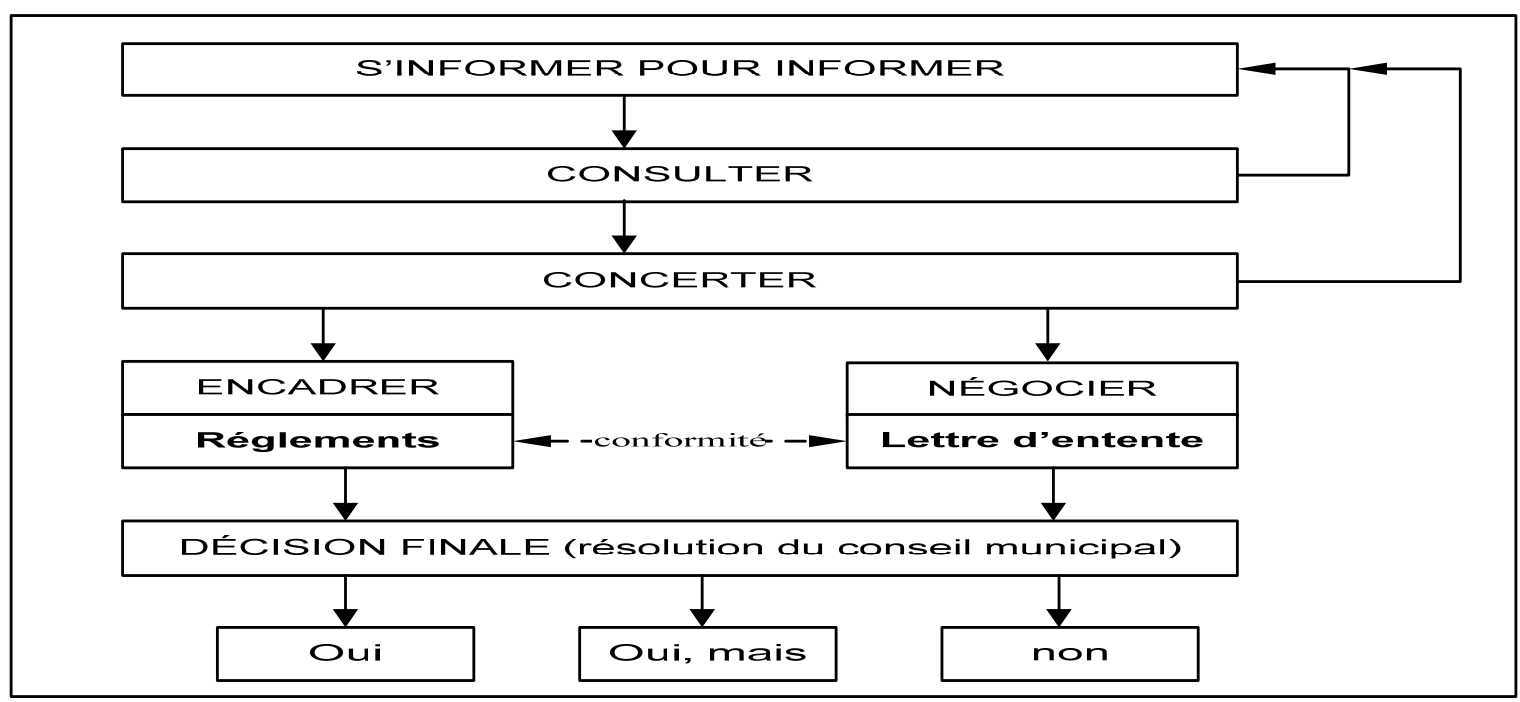




\subsection{S'informer, pour informer}

Pour prendre des décisions importantes comme celles entourant l'implantation de parcs éoliens, les élus doivent s'informer sur le sujet en général (impacts, retombées potentielles, etc.) pour être capables d'informer la population.

Il n'est pas souhaitable que les citoyens soient informés de l'existence d'un projet par les médias. Les premières démarches d'information devraient être initiées avant même l'établissement d'une tour de mesure de vent. C'est une question de respect du territoire et de la population d'accueil. Pour prendre des décisions importantes comme celles entourant l'implantation de parcs éoliens, les élus doivent s'informer sur le sujet en général (impacts, retombées potentielles, etc.) pour être capables d'informer la population. À cette fin, organiser des séances d'information publiques est une tâche importante. On commence par de l'information générale sur l'énergie éolienne pour comprendre les enjeux du développement éolien. Puis les questions deviennent de plus en plus pointues.

Quand la population aura obtenu assez d'information de la part du promoteur et des autres intervenants sur le projet spécifique, elle sera en mesure de se positionner sur celui-ci lors de séances de consultation. Cet échange d'information ${ }^{1}$ se poursuivra alors au cours des différentes étapes d'implantation d'un projet grâce à la concertation des différents acteurs impliqués dans le processus de décision ${ }^{\mathrm{e}}$. Le tableau 1 précise l'ampleur de ce rôle à assumer pour les élus.

\section{Tableau 1 : S’informer, pour informer}

Objectif : Recueillir une première série d'information pour répondre aux questions préliminaires de la population.

Moyens :

- Se renseigner auprès des institutions compétentes;

- Demander les renseignements manquants;

- Organiser des réunions d'information;

- Assigner une personne responsable du dossier éolien à la municipalité.

Conseils pratiques :

- Agir au tout début du projet;

- Répondre rapidement et clairement aux questions posées par la population;

- S'aider de plusieurs sources d'information si nécessaire, faire attention aux rumeurs;

- S'aider d'experts à chaque étape du développement;

- S'assurer d'obtenir une information complète et transparente;

- Faire attention aux tentatives de désinformation;

- Trouver le moyen de communication le mieux adapté pour renseigner la population. 


\subsection{Consulter, pour savoir quoi penser}

Les élus ne peuvent pas prendre pour acquis que la population est favorable ou défavorable à un projet de développement de la filière éolienne.

Les élus ne peuvent pas prendre pour acquis que la population est favorable ou défavorable à un projet de développement de la filière éolienne. Le but d'une démarche de consultation est de connaître les préoccupations, les attentes ou les demandes de la population concernant un projet. Cette démarche est essentielle pour une prise de décision éclairée et, le cas échéant, pourra s'avérer utile dans les négociations entre le promoteur et la municipalité pour justifier l'adoption de mesures supplémentaires d'atténuation ou de compensation des impacts. Des moyens de consultation doivent être mis en place, notamment des séances publiques où les citoyens pourront s'exprimer et être entendus (Tableau 2). Cette période de consultation doit se faire tôt dans le processus décisionnel du projet pour permettre, ultérieurement, le dialogue entre élus et promoteur, et un suivi concerté de la position de la municipalité. En matière d'éolien, on dit qu'elle devrait débuter dès l'étape d'implantation d'une tour de mesure de vent ${ }^{2}$.

\section{Tableau 2 : Consulter, pour savoir quoi penser}

Objectifs : Connaître les préoccupations, attentes et demandes de la population; identifier le compromis que la population est prête à accepter pour un développement éolien sur son territoire.

\section{Moyens :}

- Organiser des consultations publiques avec des périodes de questions;

- Organiser des forums, des débats ou des assemblées publiques;

\section{Conseils pratiques :}

- À débuter au plus tôt dans le processus décisionnel du projet;

- Se baser sur des principes de transparence pour que la position des élus soit représentative;

- Être à l'écoute de sa population, une consultation bien réalisée renforce la légitimité de la décision des élus.

\subsection{Concerter, s'entendre pour agir}

Après l'information et la consultation, la concertation des acteurs potentiellement impliqués dans les différentes étapes de développement peut se réaliser. Concerter, c'est s'entendre pour agir selon les prérogatives et la fonction de chacun (Tableau 3).

Le but est d'améliorer le projet en collaboration avec les acteurs du milieu afin de valoriser les avantages attendus des éoliennes sur le territoire d'accueil et accepter leur intégration dans la collectivité locale. C'est un élément essentiel dans la conduite et l'évolution d'un projet. Cependant, ce n'est pas non plus un gage de consensus car certains points de dissension sont de nature non négociable. Elle doit permettre de conforter la légitimité d'un projet ou d'une décision. À noter que la décision peut consister également en un refus de parc éolien si les diverses parties prenantes au processus n'arrivent pas à s'entendre. 
Tableau 3 : Concerter, s’entendre pour agir

Objectif : Définir des alternatives viables au projet à l'étude de concert avec les citoyens et les représentants de l'administration publique pour le rendre acceptable et pouvoir le concrétiser.

Moyens :

- Création d'un comité éolien de la MRC pour effectuer la planification de projets, favoriser la coexistence de plusieurs projets sur un même territoire et réduire l'impact cumulatif des projets;

- Création d'autant de comités locaux de concertation que de municipalités touchées par le projet, pour réaliser des aménagements locaux au cadre de référence adopté dans le comité de pilotage;

- Intégration possible des différents comités locaux de concertation dans un comité de pilotage du projet pour créer un cadre de référence normatif et débattre des aspects paysagers.

Conseils pratiques :

- Intéresser les acteurs locaux aux possibilités d'investissement dans le projet;

- Sensibiliser les différents groupes sociaux du territoire concerné;

- Favoriser l'appropriation des projets en encourageant l'implication des acteurs territoriaux;

- Rechercher un consensus le plus large possible sur le choix de l'aménagement;

- Donner un sens au projet.

Par ailleurs, la pratique montre qu'un projet concerté s'implante avec une relative facilité. En matière de concertation, le rôle de l'élu est essentiel car il est l'intermédiaire entre le porteur de projet et la population, et le premier interlocuteur des promoteurs et des propriétaires fonciers. Si le dialogue est déficient, ce sont les élus qui risquent d'être blâmés par la population ${ }^{1}$. Le guide propose une organisation territoriale de la concertation à plusieurs niveaux illustrés à la figure 3 . Au niveau régional ou interrégional, les comités de concertation interrégionaux cherchent à améliorer le cadre réglementaire existant (Pourrait-il y avoir des normes communes concernant les redevances des parcs? Pourrait-on envisager un développement local par des tarifs fixes?). Au niveau régional et inter-municipal, les comités éoliens régionaux cherchent à coordonner plusieurs projets sur un même territoire pour limiter les impacts cumulatifs et planifier les projets en définissant des zones d'exclusion possibles ou des aires protégées. Il s'agit aussi d'optimiser les retombées régionales des projets et de la filière. L'objectif est de garantir un développement maîtrisé de l'énergie éolienne en accord avec les réalités régionales. Les acteurs de cette concertation sont les entités régionales, les services de l'État et les différents organismes régionaux.

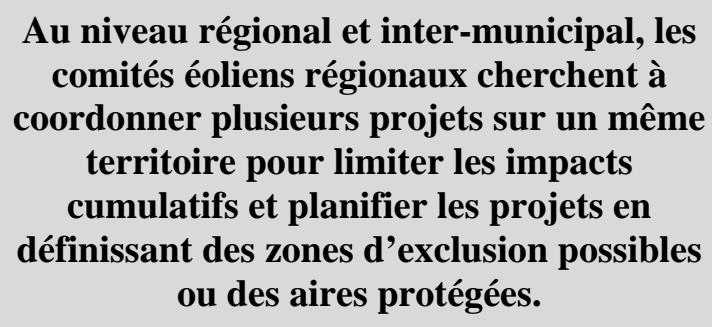

Au niveau régional et inter-municipal, les comités éoliens régionaux cherchent à coordonner plusieurs projets sur un même territoire pour limiter les impacts cumulatifs et planifier les projets en définissant des zones d'exclusion possibles ou des aires protégées. 
Figure 3 : Schéma proposé pour l’organisation de la concertation au Québec

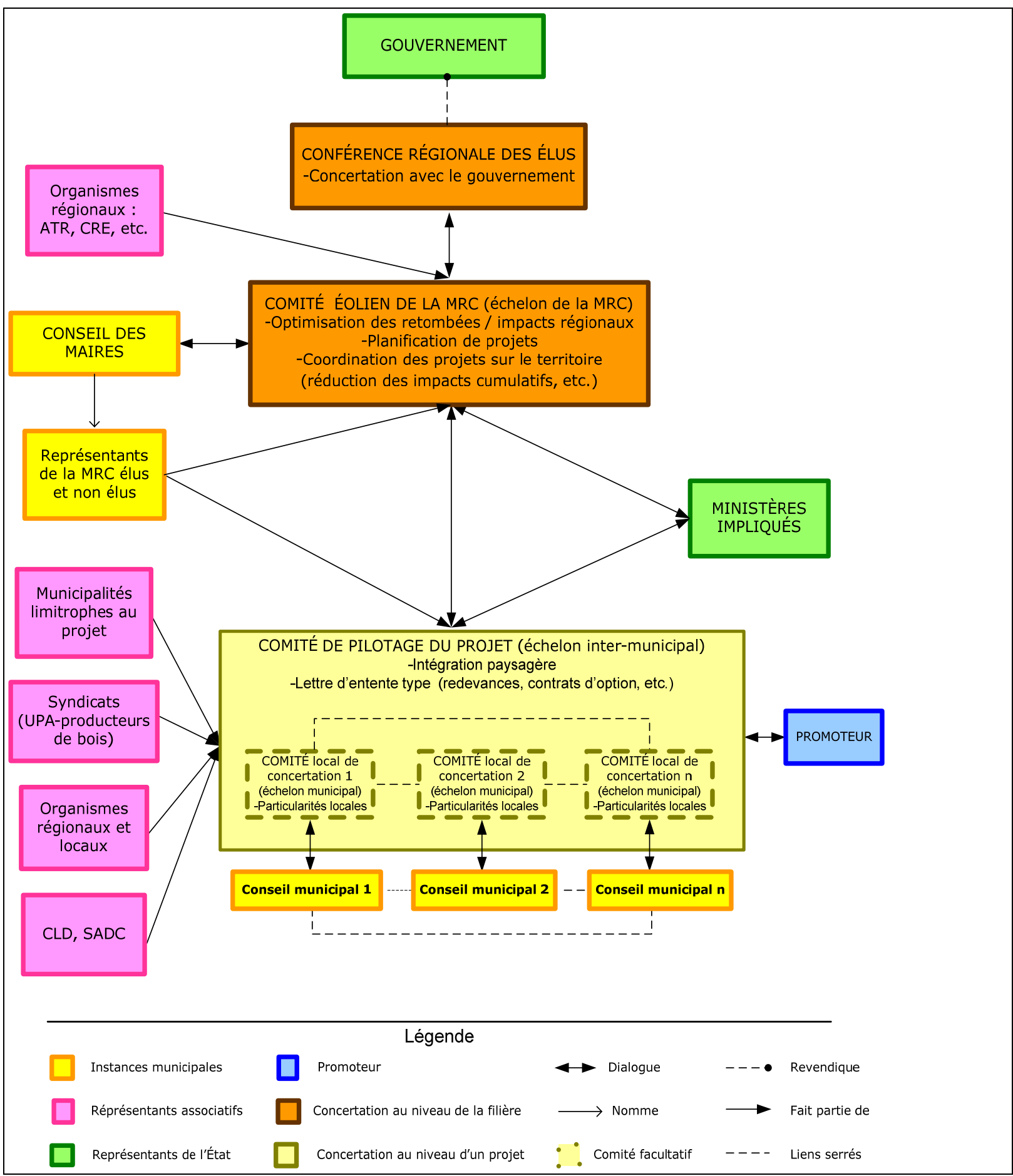


Comme le périmètre d'incidence d'un projet éolien est étendu, les impacts visuels peuvent dépasser les frontières d'une municipalité. Il est dès lors naturel

d'associer les municipalités limitrophes à la définition d'un projet qui les concerne également.

Au niveau inter-municipal et/ou municipal, le ou (les) comité(s) de pilotage ${ }^{\mathrm{f}}$ du projet vise(nt) à réaliser une concertation sur un projet spécifique. Le but est l'acceptation locale du projet éolien par l'ensemble des communautés d'accueil. Certains enjeux d'un projet spécifique devraient être débattus au niveau intermunicipal. Comme le périmètre d'incidence d'un projet éolien est étendu, les impacts visuels peuvent dépasser les frontières d'une municipalité. Il est dès lors naturel d'associer les municipalités limitrophes à la définition d'un projet qui les concerne également.

Aussi, il est nécessaire de négocier à ce niveau un cadre de référence identique sur des enjeux communs (comme les redevances aux municipalités). Le but est de maximiser les retombées économiques et de réduire les impacts équitablement. À cette concertation inter-municipale, devrait se greffer une concertation locale au sein de chaque municipalité impliquée.

La concertation locale a pour but de rendre le projet compatible avec le territoire et les attentes des habitants, chaque territoire présentant des spécificités propres. C'est à ce niveau que l'on négocierait la localisation précise des éoliennes et les mesures d'accommodement associées. Des représentants des citoyens et organismes devront être choisis pour représenter la diversité des points de vue existants.

Enfin, dès que le projet est accepté, un comité de suivi devrait être mis en place dès le début de la construction du projet et jusqu'aux premières années d'exploitation du parc. Le but est d'optimiser les retombées régionales ou locales pendant la construction du projet et d'atténuer les impacts. Par après, il faudra effectuer des suivis environnementaux légaux, comme ceux concernant le niveau de bruit ou la mortalité des oiseaux. Les membres de ce comité évolueront donc en fonction de la temporalité du projet ${ }^{1}$.

\subsection{Encadrer, pour agir dans le respect}

Le pouvoir légal des autorités locales concerne l'encadrement réglementaire et les lettres d'entente. Ces deux aspects sont essentiels pour protéger le territoire et rassurer la population. En l'absence de réglementation, le promoteur d'un projet d'énergie éolienne peut faire ce qu'il veut sur le territoire. Il est donc nécessaire d'impliquer les aménagistes le plus tôt possible dans le processus pour encadrer l'utilisation des éoliennes sur le territoire. Les élus ne peuvent agir qu'à l'intérieur des pouvoirs qui leur sont conférés par la loi. Au Québec, il n'est par exemple pas possible d'adopter des règlements qui auraient pour effet d'interdire totalement sur son territoire des activités qui sont considérées légales. Ce faisant, ils peuvent en restreindre largement l'utilisation en adoptant des normes de distance ou de bruit contraignantes. De plus, le promoteur a des obligations légales à obtenir de la part des autorités locales avant que son projet soit accepté. Par exemple, la municipalité doit lui délivrer un avis de conformité avec la réglementation municipale existante et un permis de construire, et lui accorder des droits de passage si un terrain municipal est utilisé lors de la construction. Il doit aussi s'assurer de l'approbation des autorités pour l'utilisation des routes. Finalement, c'est dans l'intérêt du promoteur d'avoir une bonne entente avec la ou les municipalités impliquées. Une lettre d'entente entre la municipalité et le promoteur facilite l'acceptabilité sociale, et donc, son approbation finale par les autorités administratives. Le tableau 4 présente le contenu de rôle d'encadrement.

Le pouvoir légal des autorités locales concerne l'encadrement réglementaire et les lettres d'entente. Ces deux aspects sont essentiels pour protéger le territoire et rassurer la population. En l'absence de réglementation, le promoteur d'un projet d'énergie éolienne peut faire ce qu'il veut sur le territoire. 
Tableau 4 : Encadrer, pour agir dans le respect

Objectif : Délimiter les zones d'implantation dans le respect de l'environnement, des citoyens et du projet.

Moyens :

- Utilisation des outils réglementaires existants : RCI au niveau des MRC et RZ, PIIA, PAE ou règlement sur le bruit au niveau des municipalités.

\section{Conseils pratiques :}

Pour l'adoption de RCI :

- S'aider des commissaires du BAPE pour réaliser une consultation publique;

- Moduler les distances réglementaires en fonction de la taille du parc;

- Prouver scientifiquement chaque contrainte d'exclusion. Se référer à des experts si nécessaire.

Pour l'adoption de règlements municipaux :

- Utiliser des outils réglementaires discrétionnaires au niveau des municipalités pour apporter plus de souplesse au RCI et plus de démocratie par une consultation de la population;

- S'entendre avec le promoteur pour partager les frais associés à la mise à niveau des règlements municipaux.

Pour l'étude d'impacts :

- Participer à la consultation préliminaire de l'étude d'impacts (coordination effectuée par le comité de pilotage, s'il existe).

\subsection{Négocier, pour optimiser les résultats}

En l'absence d'un cadre réglementaire national normé, la négociation sur les retombées collectives s'effectue directement entre le promoteur et les municipalités concernées par le projet. Elle consiste principalement en la rédaction d'une lettre d'entente signée par les deux parties. Celle-ci a pour objet de fixer le montant des redevances à verser aux municipalités, des compensations (restauration des routes en leur état d'origine, etc.), toutes les autres obligations $\mathrm{du}$ promoteur, etc. Des négociations bien menées permettent d'atteindre un compromis acceptable pour la population. Les élus visent la conclusion d'une entente avec le promoteur qui maximise les retombées locales et minimise les impacts environnementaux et sociaux des projets. Il peut être avisé de la part des municipalités locales de se regrouper et de s'entourer d'experts dans le domaine afin de modifier le rapport de force en leur faveur et d'améliorer leur pouvoir de négociation. Le résultat final des négociations peut se traduire par une résolution du conseil municipal l'autorisant à signer une lettre d'entente. Le tableau 5 précise le contenu de ce rôle de négociation en vue d'optimiser les résultats.

Les élus visent la conclusion d'une entente avec le promoteur qui maximise les retombées locales et minimise les impacts environnementaux et sociaux des projets. 
Tableau 5 : Négocier, pour optimiser les résultats

Objectif : Optimiser les retombées locales sans compromettre l'environnement et la qualité de vie des citoyens.

\section{Moyens :}

- Création d'une table de négociation avec l'ensemble des municipalités concernées si un comité de pilotage du projet n'existe pas. Sinon, ce sera les prérogatives du comité de pilotage du projet.

\section{Conseils pratiques :}

- Privilégier les négociations collectives entre les différentes municipalités concernées par le projet;

- Favoriser un dialogue constructif pour l'ensemble des parties;

- Maintenir une ouverture d'esprit réciproque;

- Assurer un leadership municipal serait souhaitable sans toutefois s'ingérer dans la négociation des contrats d'option entre le promoteur et les propriétaires fonciers;

- Faire attention aux conflits d'intérêts;

- Représenter l'ensemble des citoyens.

\section{CONCLUSION}

\section{Les élus ont des responsabilités et rôles importants en matière de développement éolien, ou plus généralement de tout développement énergétique s’implantant sur un territoire donné. Sans leur intervention, la réalisation d'un tel projet devient difficile.}

Les élus ont des responsabilités et rôles importants en matière de développement éolien, ou plus généralement de tout développement énergétique s'implantant sur un territoire donné. Sans leur intervention, la réalisation d'un tel projet devient difficile. Sans présager du devenir de chaque projet, ils peuvent en bonifier certains aspects et ils peuvent jouer un rôle de médiateur entre le promoteur et la population locale accueillant le projet. C'est l'enjeu de la gouvernance territoriale locale qui est en cause ici et qui devrait être basée sur des principes démocratiques transparents, favorisant la discussion autour des enjeux essentiels du projet. Les maîtres-mots sont alors l'information, la consultation et la concertation. Ces balises ont pour fonction d'outiller les élus pour favoriser le développement d'un projet éolien socialement acceptable. Le but n'est pas de leur dire quoi faire. Les idées sont formulées sous la forme de conseils pratiques ou de suggestions d'organisation territoriale pour faciliter et offrir des pistes d'actions concrètes sur leur territoire, qu'ils connaissent certainement mieux que n'importe quel expert. Par exemple, une proposition d'organisation d'une concertation régionale en matière d'éolien y est suggérée pour essayer de mieux comprendre le rôle de chaque échelon territorial (municipalité, MRC et Conférence régionale des élus) dans la concertation touchant un projet spécifique, la coordination de plusieurs projets au niveau régional et la concertation portant sur le développement de la filière éolienne. 
Nous avons constaté que le guide ne reste pas sur les tablettes et sert à de nombreux intervenants (des citoyens, des groupes de pression ou des élus) pour prendre de l'expérience sur la question du développement de l'énergie éolienne. Dans certains cas, il a également favorisé la mise en place d'outils institutionnels innovants. Par exemple, la Conférence régionale des élus de la GaspésieÎle-de-la-Madeleine a récemment créé une Régie inter-municipale afin de coordonner l'action des promoteurs municipaux dans la perspective des appels d'offres communautaires $^{2}$. Selon nous, il s'agit d'un pas dans la bonne direction pour s'orienter vers une coopération régionale efficace directement inspirée par le Guide.

\section{BIBLIOGRAPHIE}

1 Feurtey, É. (dir.) (2008). Énergie éolienne et acceptabilité sociale : Guide à l'intention des élus municipaux du Québec. Cap-Saint-Ignace: Conférence régionale des élus de Chaudière-Appalaches, ${ }^{2}$ Feurtey, É. et Dufour, D. (2008). Chapitre 7 «Rôles et responsabilités des élus». Dans É. Feurtey (dir.) (2008) Énergie éolienne et acceptabilité sociale : Guide à l'intention des élus municipaux du Québec, Cap-Saint-Ignace: Conférence régionale des élus de Chaudière-Appalaches, pp. 46-68. Jean, Bruno (2005). «Postface : Le projet scientifique du Centre de recherche sur le Développement territorial (CRDT) : repousser les frontières de la connaissance sur le développement territorial ». Dans Territoires et fonctions. Tome 2 B. Jean et D. Lafontaine (dir.) (2005), Rimouski: GRIDEQ et CRDT, pp. 281-295. Lavoie, A. (2010). "Les MRC gaspésiennes se donnent une Régie intermunicipale de l'énergie. » Le Pharillon. Le 23 avril 2010. Saucier, C., Côté, G., Fortin, M.-J., Jean, B., Lafontaine, D., Feurtey, É., Guillemette, M., Méthot, J.-F., et Wilson, J. (2009). Développement territorial et filière éolienne. Des installations éoliennes socialement acceptables : élaboration d'un modèle d'évaluation de projets éoliens dans une perspective de dévelop-pement territorial durable. Rapport final et résumé - Unité de recherche sur le développement territorial durable et la filière éolienne, Rimouski: Université du Québec à Rimouski, 228 p. et 10 p., accessible en ligne: http://www.uqar.qc.ca/crdt/fr/frames.html.

\section{NOTES}

${ }^{a}$ L.R.Q., c. A-19.1

${ }^{\mathrm{b}}$ Ce Guide de l'éolien origine de recherche-action qui est le résultat d'une coopération décentralisée entre des régions françaises (principalement Poitou-Charentes) et québécoises mentionnés ci-haut. Il a été rendu possible grâce à l'aide du Fonds franco-québécois de coopération décentralisée. Divers organismes ou laboratoires ont collaboré à l'une ou l'autre des parties de ce guide: la Chaire de recherche du Canada en développement régional et territorial, à la Chaire de recherche du Canada en développement rural, au Laboratoire de recherche en énergie éolienne (tous trois de l'UQAR), au Conseil régional de l'environnement du Bas-Saint-Laurent et à la Coopérative de développement régional du Bas-Saint-Laurent/Côte Nord. Il en résulte une ouvrage: Énergie éolienne et acceptabilité sociale - Guide à l'attention des Élus municipaux du Québec (Feurtey, 2008).

${ }^{c}$ Définition de l'Unité de recherche sur le développement territorial durable et la filière de l'UQAR, dans le rapport final de recherche intitulé «Développement territorial et filière éolienne. Des installations éoliennes socialement acceptables : élaboration d'un modèle d'évaluation de projets éoliens dans une perspective de développement territorial durable» (Saucier et al., 2009). Celui-ci est accessible en ligne sur le site web suivant: $\mathrm{http}: / /$ www.uqar.qc.ca/crdt/fr/frames.html (consulté le 23 juin 2010).

d Pour cette notion, voir les travaux du CRDT et notamment ceux de Bruno Jean (2005).

e L'annexe 1 constitue une liste préliminaire d'information sur le développement de l'énergie éolienne au Québec que l'on peut consulter facilement sur Internet

${ }^{\mathrm{f}}$ Il peut y avoir autant de comités locaux de concertation que de municipalités affectées par le projet. Dans ce cas-là, un Comité inter-municipal ou comité de pilotage du projet gère l'ensemble. 


\section{Annexe 1 : Information disponible au Québec sur le développement de l'énergie éolienne}

\section{- Auprès des différents ministères et organismes étatiques :}

- Le MAMROT pour la réalisation des réglementations locales, la possibilité de participer financièrement aux projets, etc. Il existe plus de 10 fiches d'information sur l'énergie éolienne :

http://www.mamr.gouv.qc.ca/amenagement/amen_amen_eoli.asp\#orientations (consulté le 31 mai 2010).

- Le MNRF pour les politiques d'attribution des contrats en territoire public et la stratégie énergétique du Québec en matière d'énergie :

http:/www.mrnf.gouv.qc.ca/energie/eolien/index.jsp (consulté le 31mai 2010).

http://www.mrnf.gouv.qc.ca/energie/strategie/ (consulté le 31 mai 2010).

- HQ pour voir les appels d'offres en cours, obtenir la liste des projets implantés ou comprendre les bases de l'éolien :

http://www.hydroquebec.com/distribution/fr/marchequebecois/index.html (consulté le 31 mai 2010).

http://www.hydroquebec.com/distribution/fr/marchequebecois/parc eoliens.html (consulté le 31 mai 2010).

http://www.hydroquebec.com/comprendre/eolienne/index.html (consulté le 31 mai 2010).

- La Régie de l'énergie sur le contexte réglementaire en matière de tarification :

http://www.regie-energie.qc.ca/audiences/en cours.html (consulté le 31 mai 2010).

- Le site du BAPE pour les évaluations environnementales sur les projets déjà réalisés et les mandats en cours d'évaluation, et toute autre information sur les impacts environnementaux des projets éoliens :

http://www.bape.gouv.qc.ca/sections/mandats/themes/ma_eoliennes.htm (consulté le 31 mai 2010).

- Les différents guides sur l’éolien réalisés à ce jour (hormis ceux du gouvernement québécois) :

- Le guide du Conseil régional de l'environnement du Bas-Saint-Laurent :

http://www.crebsl.com/media/Rubrique\%20B-10 ENERGIE/PDF/11 GuideFiliereEolienne.pdf (consulté le 31 mai 2010).

- Les guides éoliens français, disponibles sur le site Web éolien de la région Poitou-Charentes : http://www.eolien-poitou-charentes.com/download_list_5.php (consulté le 31 mai 2010).

- Sur l'énergie éolienne :

- Bible de référence sur l'énergie éolienne, la Danish Wind Industry Association (windpower.org) : http://guidedtour.windpower.org/fr/tour/wres/index.htm (consulté le 31 mai 2010).

- Lien vers l'ensemble des associations nationales pour l'énergie éolienne : http://www.talentfactory.dk/composite-224.htm (consulté le 31 mai 2010).

- L’éolien au Québec (Site Web du TechnoCentre éolien) : https://www.eolien.qc.ca/ (consulté le 31 mai 2010).

- L'éolien au Canada (CANWEA) : http://www.canwea.ca/index f.php (consulté le 31 mai 2010).

- L'éolien en France (ADEME) : Ademe-éolien, http://www.suivi-eolien.fr/ (consulté le 31 mai 2010).

- L'éolien en Europe (EWEA) : http://www.ewea.org/ (consulté le 31 mai 2008).

- Base de données sur les éoliennes et les parcs éoliens : http://www.thewindpower.net/ (consulté le 26 mai 2010).

- Livres sur l'éolien :

- Saulnier, Bernard et Réal Reid (2009). L'éolien au cœur de l'incontournable révolution énergétique. Québec: Éditions Multimondes, 396 p., disponible sur le site web : http://multim.com/titre/?ID=280 (consulté le 31 mai 2010).

- Bouchard, Roméo (dir.) (2007). L'éolien au Québec, pour qui souffle le vent? Actuels, Montréal: Écosociétés, 126 p., disponible sur le site web : http://www.ecosociete.org/t109.php (consulté le 31 mai 2010).

- Gipe, Paul (2006). Le grand livre de l'éolien. Paris: Observ'ER, 516 p., disponible sur son site web: http://www.wind-works.org/books/index.html (consulté le 31 mai 2010). 